\title{
The biology and therapy of brain metastasis: targeting the astrocytes
}

\author{
Isaiah J. Fidler, D.V.M., Ph.D. \\ Department of Cancer Biology, Metastasis Research \\ Laboratory, The University of Texas M. D. Anderson \\ Cancer Center, Houston, Texas, USA
}

Despite improvements in surgical techniques, general patient care, and local and systemic adjuvant therapies, most deaths from cancer still result from the progressive growth of metastases that are resistant to conventional therapies. The outcome of metastasis depends on multiple interactions of metastatic cells ("seed") with homeostatic mechanisms ("soil") which tumor cells can exploit. A clear example of these interactions is the biology of brain metastasis.

More than $40 \%$ of cancer patients succumb to brain metastasis, specifically nearly $50 \%$ of patients with lung cancer, $30 \%$ of patients with breast cancer, and $20 \%$ of patients with melanoma. With improved local control and therapy of metastasis to visceral organs, the morbidity and mortality due to late diagnosed brain metastasis is projected to rise. The median survival for untreated patients is $1-$ 2 months, which may be extended to 6 months with surgery, conventional radiotherapy, and chemotherapy.

The brain microenvironment regulates the resistance of tumor cells to systemic therapy. The mechanism for this resistance has been attributed to the blood-brain barrier (BBB), which protects brain cells from circulating toxic substances. In brain metastases, however, the BBB is permeable due to increased production of VEGF/VPF by tumor cells. Hence, resistance of brain metastases to chemotherapy is mediated by a different mechanism.
Histologic examination of clinical specimens of brain metastases reveals that the lesions are densely surrounded and infiltrated by activated astrocytes expressing glial fibrillary acidic protein (GFAP). GFAP-positive astrocytes are also associated with experimental brain metastases produced by orthotopic implantation of metastatic cells. We isolated astrocytes from the brain of the "ImmortoMouse" and established the cells in culture. Astrocytes cocultured with human melanoma, breast cancer, or lung cancer cells protected the tumor cells from all tested chemotherapeutic agents (taxol, VCR, VBL, ADR, cisplatinum, 5-FU). Establishment of a gap junction communication between the astrocytes and tumor cells was required for this protection. Coculture of tumor cells with other tumor cells or with fibroblasts did not protect the cells from any chemotherapeutic drugs. Microarray experiments for cross-species hybridization (human tumor cells cocultured with mouse astrocytes or mouse fibroblasts) identified upregulation of several survival genes, including GSTA5, BCL2-L1, and TWIST1, in tumor cells cocultured with astrocytes, but not with fibroblasts. Knocking down these genes with specific SiRNA rendered the tumor cells sensitive to chemotherapy. Once removed from astrocytes, the expression of these genes declined, and the tumor cells lost the resistance to the chemotherapeutic drugs. Immunohistochemical staining of clinical specimen identified the expression of these genes in brain metastases. These data demonstrate that astrocytes influence the biological behavior of tumor cells in brain metastases and reinforce the conclusion that the organ microenvironment must be taken into consideration for the design of effective therapy. 